R. J. Cohen and W. T. Sullivan, III, eds.

\title{
Radio Interference and Ejecting Techniques at Beijing Astronomical Observatory ${ }^{1}$
}

\author{
X. Zhang ${ }^{2}$, T. Piao, B. Peng ${ }^{2}$, X. Wang \\ Beijing Astronomical Observatory, CAS, Beijing 100012, China \\ National Astronomical Observatories, CAS, Beijing 100012, China \\ E-mail: zxz@class1.bao.ac.cn
}

\begin{abstract}
In this paper, we first describe the situation of radio interference at the Miyun station in Beijing Astronomical Observatory, and then new developments in both hardware and software techniques of interference rejection for the Miyun Synthesis Radio Telescope (MSRT) are described.
\end{abstract}

\section{Introduction}

The Miyun Synthesis Radio Telescope (MSRT) was built in 1967 on the north bank of the Miyun reservoir, about $120 \mathrm{~km}$ from the Beijing city. There was a very quiet radio environment at that time, at its operating frequency of $232 \mathrm{MHz}$. But radio interference has become very serious as the economy has developed. In the 1980s radar and taxi calling telephones were the main interference sources. Later on, TV transfer stations, cellular telephones, water and electricity power monitoring systems, PCs, and so on became new manmade radio interference sources. In 1994, due to the very serious interference, the MSRT had to stop its daily observations. While the MSRT staff appealed to the National Radio Management Committee of China to solve the problem, some anit-interference hardware and software were developed. One attempt is that 28 surface-acousticwave filters (SAWFs) were added to the telescope to reduce the effects of bad interference at $234.5 \mathrm{MHz}$. Although it is just out of the protected frequency band, it is so strong that it produced big trouble in the telescope receiver. The properties of the SAW filter and some examples of interference are demonstrated in this paper.

For interference within the MSRT band, software was developed to eject the interference from the observational data so that a much better map can be obtained. The software was designed based on the differences of phase and amplitude properties between interference and astronomical objects. Some results before and after using the software are presented.

\footnotetext{
${ }^{1}$ The project is supported by the National Natural Science Foundation of China and the National Climbing programme of China.
}

${ }^{2}$ Visiting Astronomer, MPI Bonn, Germany 


\section{New Technical Developments on Interference Rejection}

The working frequency of the MSRT is $232 \mathrm{MHz}$ and its bandwidth at the 3 -dB level is $1.5 \mathrm{MHz}$, i.e. from 231.25 to $232.75 \mathrm{MHz}$. In the $1990 \mathrm{~s}$ after the problems from radar and car-calling-telephone systems were solved, strong interference then was caused by telemetry services for flood, earthquake and electricity monitoring. Among them the worst interference, from the electricity monitoring system, is about $30 \mathrm{~dB}$ stronger than the receiver system level at 234.5 MHz. In addition to this, other interferences are from inside the telescope control room: (a) the antenna-control-computer gave interference about $10 \mathrm{~dB}$ higher than the system background noise at $236.28 \mathrm{MHz}$; (b) the correlation shell of the receiver radiated on average 7-8 dB high interference in the frequency range from 235 to $236 \mathrm{MHz}$; (c) from the GPS clock system at frequencies 230.63 and $235.5 \mathrm{MHz}$, about $10 \mathrm{~dB}$ high interference signals were found. To eliminate these interference effects, new hardware and software techniques were developed.

\subsection{Hardware Techniques}

We have two kinds of hardware techniques to constrain the interference. 1) For interference from the PCs, we simply jump the PC-bus-frequency so that its harmonic frequencies will be far from the telescope band. 2) Facing the more serious interference problems, new SAWFs were developed and added to the telescope. The good properties of SAWFs, especially the very steep passband, helps to constrain the strongest interference at $234.5 \mathrm{MHz}$ which is just out of the telescope working band. The left-hand plot in Fig. 1 shows the passband of a SAWF and in contrast the passband of the original filter is shown in the right-hand plot in Fig. 1. Table 1 lists the related numbers and shows clearly the superior anti-interference capability of the new SAWF. The main parameters of the SAWF include: flat passband of $\sim 2 \mathrm{MHz}$, shape factor $\sim 1.7$, passband ripple $\sim 0.2 \mathrm{~dB}$, noise factor $\sim 6.5 \mathrm{~dB}$ and output dynamic range $\sim 0 \mathrm{dBm}$.

Table 1. Interference at Miyun station: a comparison of the antiinterference capacity of the new and old systems.

\begin{tabular}{ccccccc}
\hline Frequency(MHz) & 229.10 & 230.63 & 232.0 & 234.5 & $235-236$ & 236.28 \\
Interf. source & PC & PC & $?$ & moni. sys. & GPS clock & PC \\
NEW & -41.7 & -20 & 0 & -43 & $<-45$ & $<-45$ \\
OLD & -5.0 & -1.0 & 0 & -2.0 & $-10 /-20$ & -20 \\
\hline
\end{tabular}

\subsection{Software Techniques}

For interference originating just outside our operating band, the hardware technique mentioned above is very effective. But hardware techniques cannot deal with interference within our band. To solve this problem two software techniques have been developed since 1989 (Yang et al. 1990), but new interference appeared after 1989, and new functions and features have been added to the original software in recent years.

Our new software design is based on understanding the observational data. The relevant data properties are: 

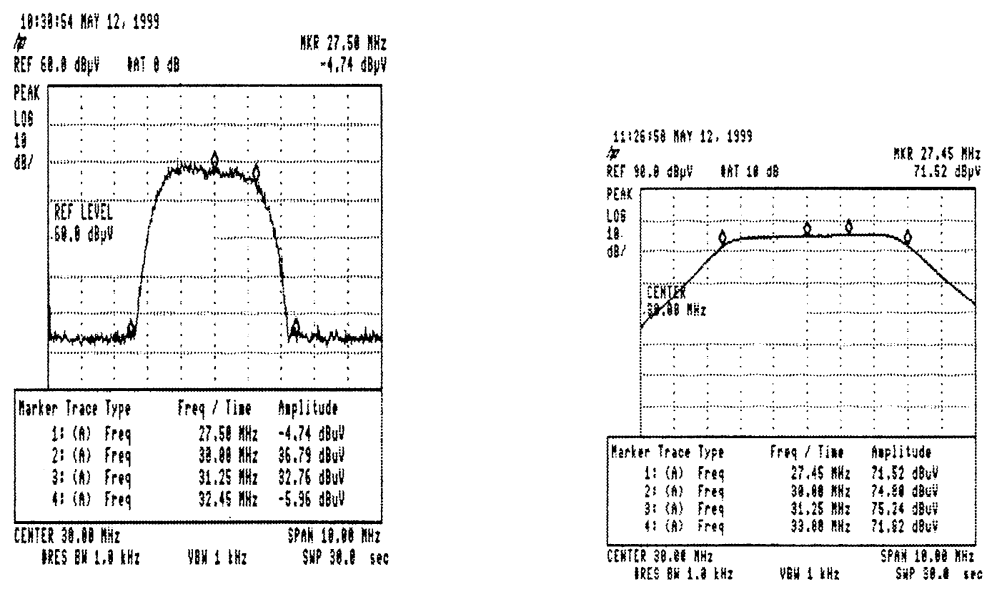

Figure 1. The left-hand plot shows the passband of a SAWF and the right the original passband

1) It appears like random noise.

2) For most sky area, signal is much lower than the telescope system noise.

3) For a signal from a radio source, phase and amplitude variation can be calculated.

4) For interference with high intensity and short duration, its effects on the map plane are long, narrow and straight lines. The interference can be simply edited out.

5) An important case is that of low level interference of long duration and varying in phase and amplitude. Its effects on the map plane could be very complex. 6) Receiver zero-offset is a special interference. It causes a series of circles of varying amplitude and radius, centred near the North Celestial Pole.

Based on the knowldge mentioned above, the new software was designed and then was used sucessfully for the Miyun Radio Sources Survey (X. Zhang el al. 1997). Fig. 2 gives an example of the recorded data before and after the interference was rejected.

\section{Results}

These new developements in both hardware and software make the MSRT run successfully. Fig. 3 shows the sky maps before and after the new software has been applied to the observing data. Its improvement to the map quality can be easily seen. 

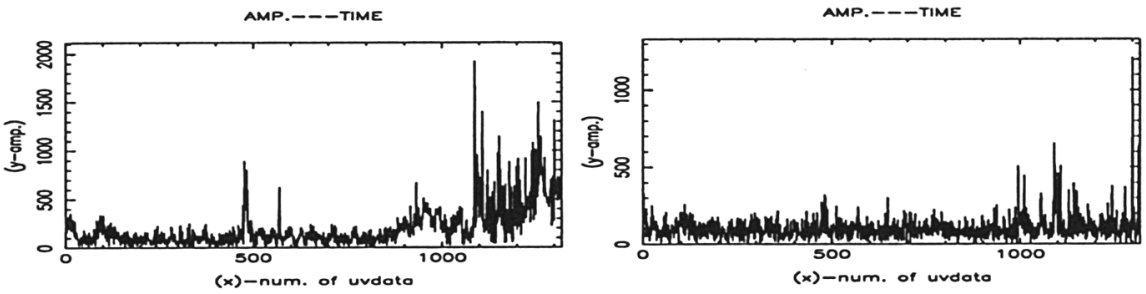

Figure 2. The two diagrams show examples of MSRT data before (left) and after (right) interference was rejected using software techniques.
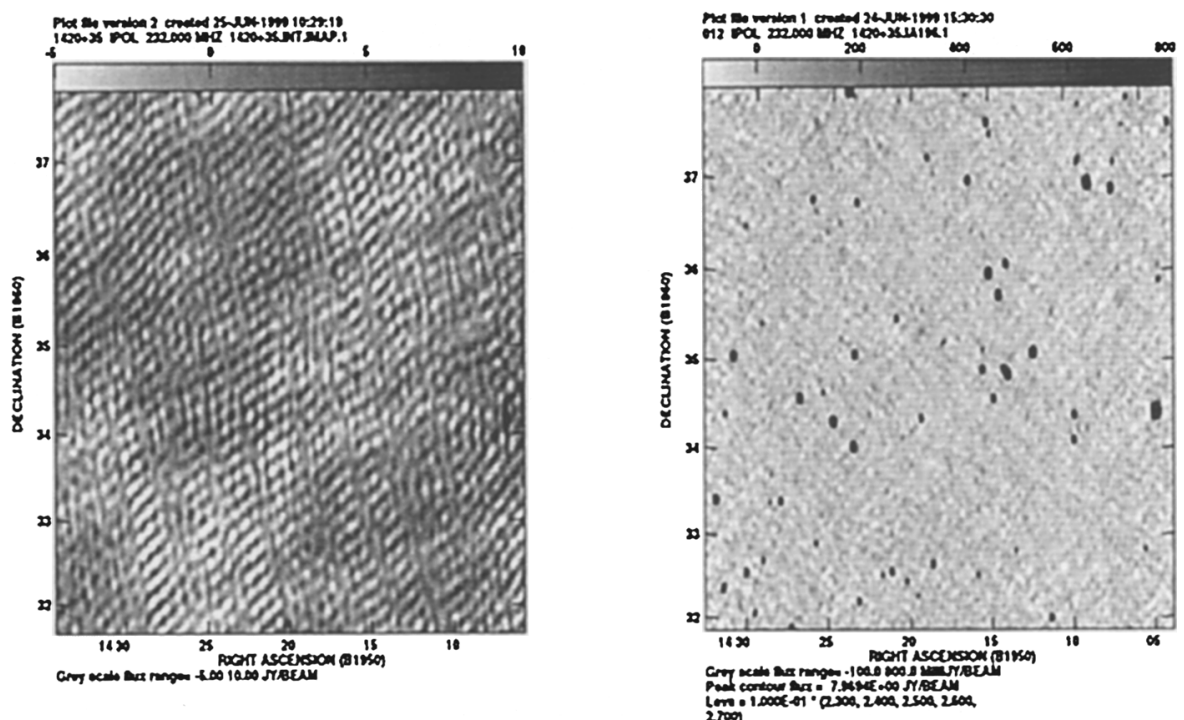

Figure 3. The left and right are sky maps before and after interference was rejected.

\section{References}

Yang, Y.P., Zheng, Y.J., Zhang, X.Z., et al., 1990, Proceedings of IAU/URSI Symp. on Radio Astronomical Seeing, editors: J.E. Baldwin and S. G. Wang, (Pergamon Press, Oxford), pp.249-254

X. Zhang, Y. Zheng, H. Chen, et al., 1997, A\&AS, .121, 59 